\title{
Suppression of Inflammatory Mucosal Milieu by Administration of Regulatory Dendritic Cells in an Animal Model of Primary Biliary Cirrhosis
}

\author{
Sheikh Mohammad Fazle Akbar, Shiyi Chen, Mamun Al-Mahtab, Masanori Abe, Osamu Yoshida
}

Yoshio Ikeda, Yoichi Hiasa, Morikazu Onji

\begin{abstract}
Background/Aim: Primary biliary cirrhosis (PBC) is an autoimmune disease of the liver for which a curative therapy is still lacking. The aim of this preclinical study was to assess if down regulation of host immunity by regulatory dendritic cells (DC) bears therapeutic implications in a murine model of PBC.

Methods: An animal model of PBC was established by injecting $5 \mathrm{mg} / \mathrm{kg}$ polyinosinic polycytidylic acid (poly I:C) twice a week in female C57BL/6 mice. Regulatory DC were produced by culturing bone marrow DC with interleukin-10 and lipopolysaccharides without or with pyruvate dehydrogenase complex (PDC, $1 \mu \mathrm{g} / \mathrm{ml}$ ). Regulatory DC and PDC-pulsed regulatory DC were injected intraperitoneally trice $(8,12$ and 20 weeks after starting of poly I:C administration) to PBC model mice. Antimitochondrial antibody (AMA) was checked in the sera and liver histology was assessed to evaluate the effect of regulatory DC on inflammatory hepatic mucosal milieu.
\end{abstract}

Results: AMA in the sera and progressive infiltration of mononuclear cells were detected in all C57BL/6 due to administration of poly I:C. Injection of regulatory DC or PDCpulsed regulatory $D C$ for 3 times caused significant reduction of infiltrating mononuclear cells in 4 of 5 PBC model mice. However, the effect of regulatory DC on AMA negativity was not documented in murine model of PBC.

Conclusion: This pilot study inspires optimisms that regulatory DC may be an immune therapeutic approach for treating PBC, however, further study about nature of antigens, dose of antigens, duration of therapy and protocol of administration of regulatory DC need further analyses.

Abbreviations: PBC: Primary biliary cirrhosis; PDC: Pyruvate dehydrogenase complex; DC: Dendritic cells; Poly I:C: polyinosinic-polycytidylic acid; AMA: Antimitochondrial antibody; UDCA: Ursodeoxycholic acid; PBS: Phosphate-buffered saline; GM-CSF: Granulocyte-macrophage colony stimulating factor; ANA: Antinuclear antibody; BM: Bone marrow; LPS: lipopolysaccharides; IL: Interleukin.

Keywords: Primary biliary cirrhosis, Regulatory dendritic cells, Murine model, AMA

How to cite this article: Akbar SMF, Chen S, Al-Mahtab M, Abe M, Yoshida O, Ikeda Y, Hiasa Y, Onji M. Suppression of Inflammatory Mucosal Milieu by Administration of Regulatory Dendritic Cells in an Animal Model of Primary Biliary Cirrhosis. Euroasian J Hepato-Gastroenterol 2012;2(1):30-34.

Source of support: Nil

Conflict of interest: None declared

\section{INTRODUCTION}

Primary biliary cirrhosis (PBC) is a chronic progressive inflammatory liver disease that leads to the immune- mediated destruction of small and medium-size intrahepatic bile duct, progressive cholestasis and eventually, fibrosis and cirrhosis of the liver. ${ }^{1-3}$ The serologic hallmark of PBC is the presence of high-titer serum antimitochondrial autoantibodies (AMA), although the role of AMA in the pathogenesis and progression of $\mathrm{PBC}$ is still elusive. ${ }^{4}$ There is no curative therapy for $\mathrm{PBC}$, although ursodeoxycholic acid (UDCA) is now widely used to treat patients with PBC. 5,6 A long-term follow-up study in a cohort of 225 patients reported 10-year survival without liver transplantation to be significantly higher in UDCA-treated patients compared with survival predicted by the Mayo model. ${ }^{7}$ However, a meta-analysis of 11 randomized trials could not confirm a significant effect of UDCA on survival and incidence of liver transplantation. ${ }^{8}$ The peroxisome proliferator-activated receptor $\alpha$-agonist, bezafibrate, has been reported to improve serum liver tests in $\mathrm{PBC}$ and this drug should undergo more extensive evaluation in patients with PBC. ${ }^{9}$ In addition, corticosteroids and other immunosuppressive agents have been evaluated for therapeutic use in PBC, however, these drugs have not been shown to markedly improve the natural course of the disease or were associated with significant toxicity during long-term treatment. ${ }^{3}$ Taken together, new and innovative therapy should be developed for PBC.

In this regard, we provided attention to immune therapy as $\mathrm{PBC}$ is an immune-mediated disease. Dendritic cells (DC), the most potent antigen-presenting cell, have recently used either to upregulate or down regulate host immunity in various pathological conditions and some of these approaches seem to have therapeutic potential. ${ }^{10}$ As an inflammatory microenvironment prevails in the liver of PBC patients, we assumed that regulatory DC that down regulate host immunity may be a choice of therapy for $\mathrm{PBC}$. In fact, regulatory DC has been used in different animal models of human diseases with potential therapeutic promises. ${ }^{11-18}$

In this study, we checked the therapeutic efficacy of regulatory DC and antigen-specific regulatory DC in an animal model of PBC. The outcome of this pilot study may be used for development of better therapeutic regimen for $\mathrm{PBC}$ and other autoimmune diseases. 


\section{MATERIALS AND METHODS}

\section{Murine Model of PBC}

An animal model of $\mathrm{PBC}$ was prepared by injecting $5 \mathrm{mg} / \mathrm{kg}$ of polyinosinic-polycytidylic acid (poly I:C, Sigma, St. Lous, MO, USA) twice a week in female C57BL/6 mice, exactly as described by us in a previous communication. ${ }^{19}$ Control mice received $200 \mu 1$ of phosphate-buffered saline (PBS). They were maintained separately at the animal house of Ehime University, School of Medicine, Ehime, Japan, under controlled conditions $\left(22^{\circ} \mathrm{C}, 55 \%\right.$ humidity, and 12 -hour day/night), and were fed freely supplied standard laboratory chow and water. All animals received adequate humane care according to good laboratory practice guidelines. The Committee of Animal Experimentation, Ehime University School of Medicine, Japan approved this study protocol.

\section{Histopathology and Immunohistochemical Examination}

To assess the extent of infiltration of inflammatory mononuclear cells, formalin-fixed, paraffin-embedded liver tissues were used for histological evaluation. The area of the liver specimens, the number of portal tracts, and the extent of cell infiltration were estimated using a combination of a digital camera and imaging tools, as described. ${ }^{19}$

\section{Detection of Autoantibodies}

The existence of autoantibodies was examined in HEp-2 cells using the commercially available fluoro HEPANA Test system (MBL, Nagoya, JAPAN), exactly as described. ${ }^{20}$ After reacting HEp-2 cells with sera, Alexa Fluor 555conjugated polyclonal goat antimouse immunoglobulin (Molecular Probes Inc. Eugene, OR) was used as a secondary antibody. Based on staining pattern, antinuclear antibody (ANA), AMA, and both ANA and AMA were detected in various sera from mice.

\section{Production of Regulatory DC}

Immature bone marrow (BM)-derived DC were prepared by culturing bone marrow cells with murine granulocytemacrophage colony stimulating factor (GM-CSF, $5 \mathrm{ng} / \mathrm{ml}$, R\&D systems Inc, Minneapolis, MN, USA) and murine interleukin (IL)-4 (5 ng/ml, R\&D systems Inc) for 7 days. ${ }^{11}$ To prepare regulatory DC, bone marrow DC were cultured with lipopolisaccharides (LPS, $1 \mu \mathrm{g} / \mathrm{ml}$, InvitroGen, Carlasbad, CA, USA) and IL-10 (20 ng/ml, R\&D systems Inc) in RPMI 1640 plus $10 \%$ fetal calf serum for 48 hours. In some studies, PDC-pulsed regulatory DC were prepared by culturing bone marrow DC with LPS, IL-10 and PDC
(1 $\mu \mathrm{g} / \mathrm{ml}$, Sigma). The cells retrieved and washed in PBS before administration to mice.

\section{Therapeutic Protocol}

Production of animal model of $\mathrm{PBC}$ and therapeutic regimen have been shown in Figure 1. Several female C57BL/6 were injected with poly I:C, twice in a week for 28 weeks. Some of the poly I:C-injected mice received regulatory DC or PDC-pulsed regulatory DC for 3 times $(8,12$ and 20 weeks after commencement of poly I:C administration). Sera were collected from all mice at 16 and 28 weeks. Mice were also killed at 16 and 28 weeks after starting poly I:C administration to assess the extent of liver damages.

\section{RESULTS}

\section{Mice Model of PBC}

A mice model of PBC was developed due to administration of poly I:C for 8 weeks and the extent of liver damages progressed along with time. As shown in Figures $2 \mathrm{~A}$ to $\mathrm{C}$, infiltration of mononuclear cells were observed around portal area (Fig. 2A), around biliary epithelia (Fig. 2B) and in hepatic parenchyma (Fig. 2C) at 16 weeks after commencing administration of poly I:C in female C57BL/6 mice. In addition, AMA and ANA were found in the sera of all mice injected with poly I:C (Figs 3A to C).

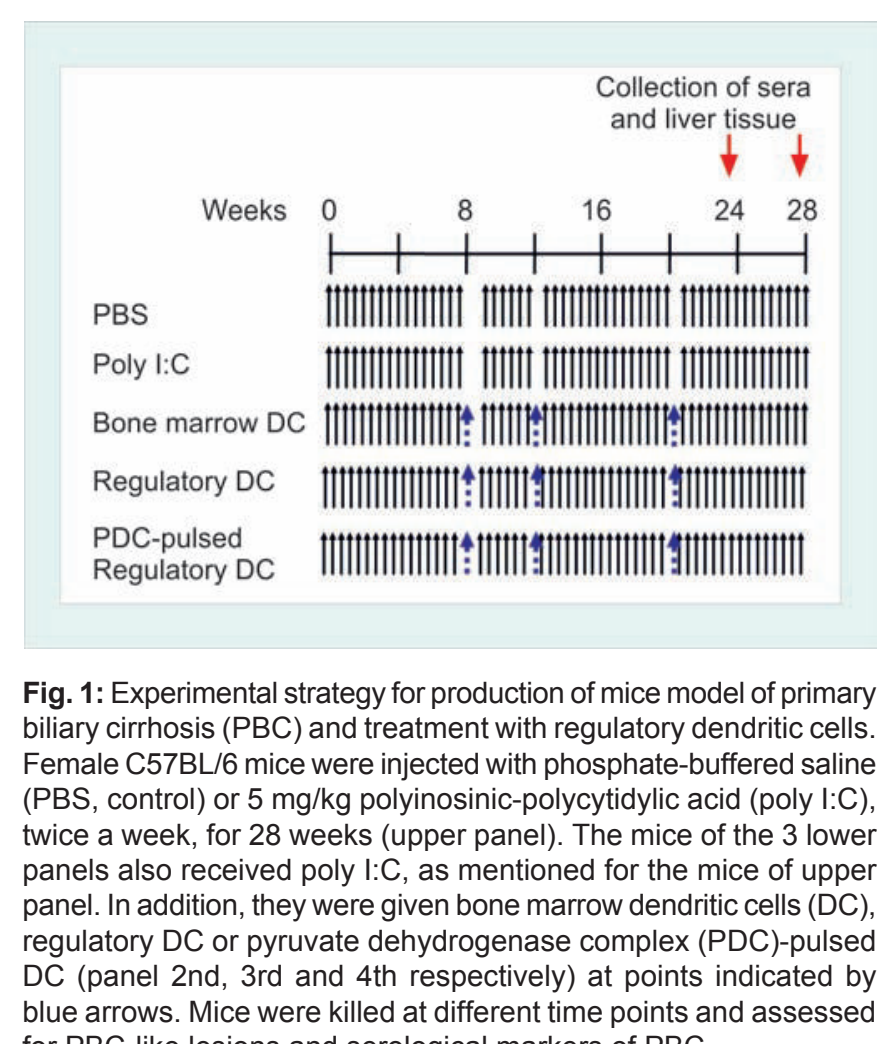
for PBC-like lesions and serological markers of PBC 


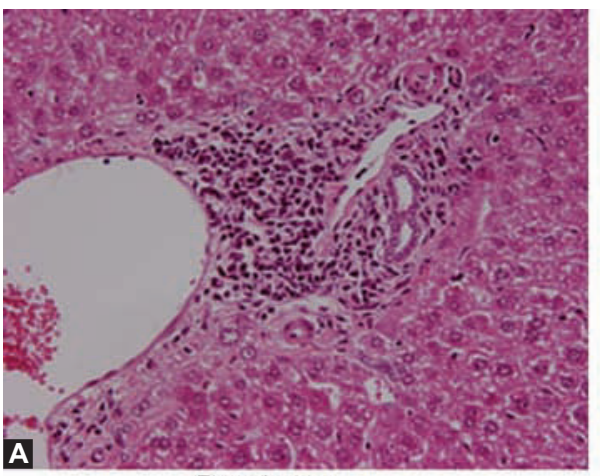

Portal area

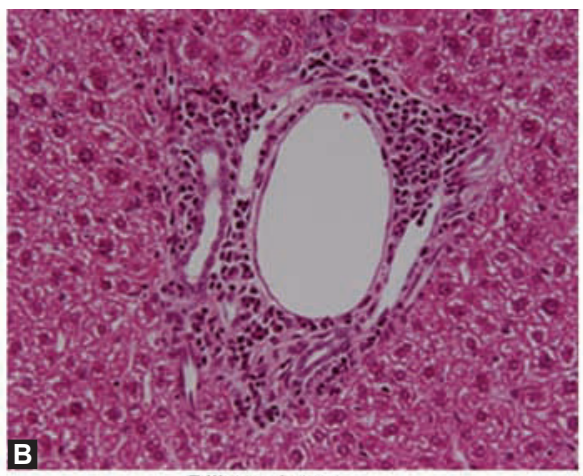

Biliary ducts

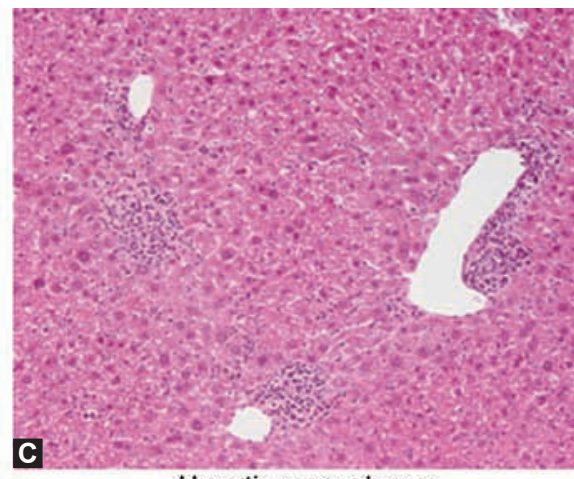

Hepatic parenchyma

Figs $2 A$ to $C$ : Features of PBC mice model due to injection with poly I:C for 16 weeks. Moderate-to-severe degrees of infiltration of mononuclear cells were seen in portal area, (A) around biliary ducts, $(B)$ and in liver parenchyma, $(C)$ due to injection with poly I:C

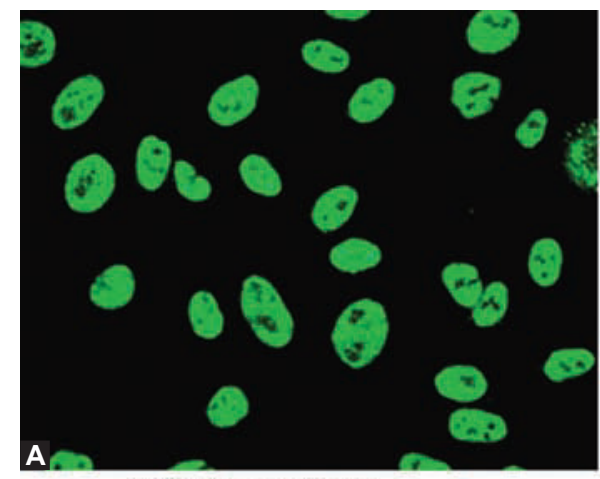

Antinuclear antibody

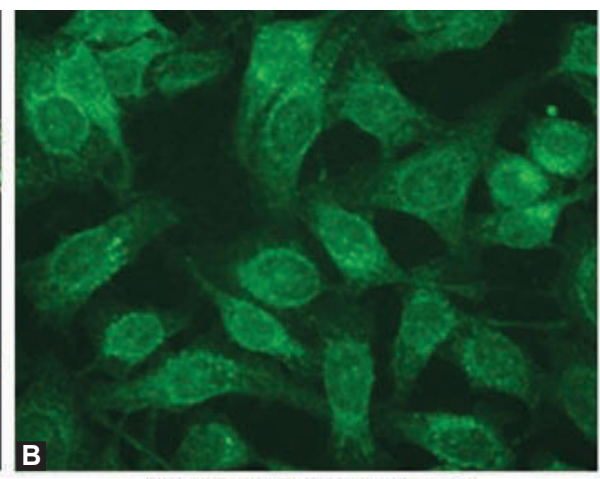

Antimitochondrial antibody

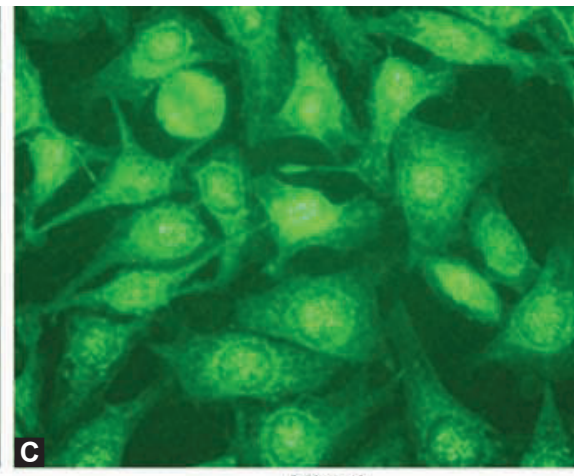

Mixed

Figs 3A to C: Presence of autoantibodies in the sera of mice injected with poly I:C. HEp-2 cells were incubated with the sera from mice injected with poly I:C. The presence of antinuclear antibodies (A) anticytoplasmic antibodies, (B) and coexistence of both antibodies, (C) was evaluated by an immunofluorescence method using Alexa Fluor 555 conjugated polyclonal antimouse immunoglobulin antibody. A representative staining pattern of autoantibodies from the sera of female C57BL/6 mice-injected with poly I:C is shown

\section{IL-10 Production by PDC-pulsed Regulatory DC}

Almost no IL-10 was produced by bone marrow DC in spontaneous culture. However, abundant amounts of IL-10 $(>1000 \mathrm{pg} / \mathrm{ml})$ were produced by regulatory DC and PDCpulsed regulatory DC in the supernatant of all cultures.

\section{Role of Regulatory DC on Inflammatory Mucosal Milieu of Animal Model of PBC}

Three administrations of regulatory DC and PDC-pulsed regulatory DC caused significantly reduced infiltration of inflammatory cells in 4 of 5 PBC model mice. The extent of infiltration of mononuclear cells of representative $\mathrm{PBC}$ model mice has been shown in Figure 4.

We did not mark any significant difference about cell infiltration in $\mathrm{PBC}$ mice due to administration of regulatory DC or PDC-pulsed regulatory DC (data not shown).

\section{Role of Regulatory DC on Serum AMA Levels}

AMA was seen in PBC model mice due to when these were examined on 16 and 28 weeks after start of poly I:C administration. Injection with regulatory DC and PDC- pulsed regulatory DC could not cause negativity of AMA in any mice.

\section{DISCUSSION}

As present understandings about pathogenesis of chronic liver diseases are broadening, it seems that new and innovative types of therapeutic approaches would be required for containment of these pathological conditions. Immune therapy represents a novel and evidence-based type therapy in this context. This is especially true for autoimmune liver diseases because there is no curative therapy for these lesions. Almost of types of therapy for PBC is endowed with limited clinical efficacy.

In this study, we have shown that regulatory DC that is capable of inducing immune tolerance in vivo may be an alternate therapeutic approach against PBC. In fact, we have already found that $\mathrm{DC}$ are involved in variable manners in the pathogenesis of PBC. Yamamoto et al from our laboratory have shown that DC in PBC patients show impaired functional capacity that may be related to breakdown of self tolerance in PBC. ${ }^{21}$ On the other hand, study from our laboratory has also shown that the anti- 

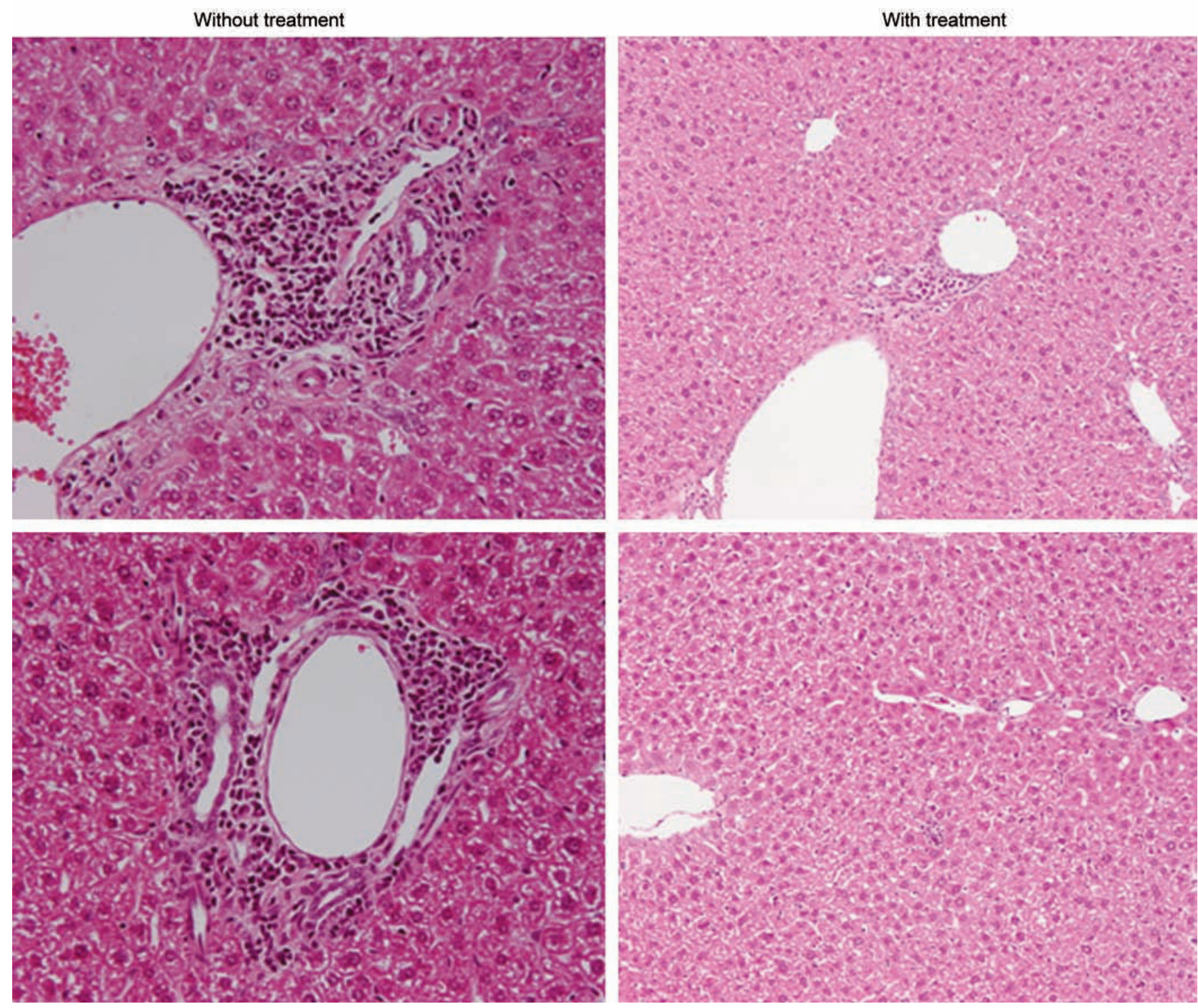

Fig. 4: Anti-inflammatory effects of regulatory dendritic cells in mice model of PBC. Accumulation of inflammatory cells in the portal area and around biliary tracts in PBC murine model of PBC. Significant down regulation of inflammatory cells due to treatment with regulatory DC. Without therapy: Receiving poly I:C according to the protocol in Figure 1. After therapy: 28 weeks receiving poly I:C according to protocol of Figure 1 with three administrations of regulatory DC

inflammatory property of bezafibrate in $\mathrm{PBC}$ is mediated through impaired anti-inflammatory action of DC. ${ }^{22}$

Administration of regulatory DC and PDC-pulsed regulatory DC but not BM DC caused reduced accumulation of inflammatory cells in the portal area and hepatic parenchyma. However, a significant improved capacity of PDC-pulsed DC over unpulsed regulatory DC could not be substantiated in this study. There may be several reasons for this. PDC represents a PBC-related antigen because almost all patients with $\mathrm{PBC}$ show antibody to PDC or its epitopes, ${ }^{23}$ even if they are AMA-negative. PDC-specific antibody has shown diagnostic utility in PBC. But, PDC may not be a target antigen of immune-mediated damages of PBC. Thus, future study using other PBC-related antigen pulsed regulatory DC may be a better therapeutic choice. In addition, we have used only one dose of PDC and kinetics of DC numbers for therapeutic effects in murine PBC model has not been explored. Studies would be required to assess other PBC-related antigens and different doses of PDC and DC to prepare antigen-specific regulatory DC for therapeutic purposes. However, treatment with regulatory DC clearly showed their anti-inflammatory activities, but, the levels of AMA negativity could not be attained by DC-based immune therapy. Although little is known about genesis of AMA and inflammatory microenvironment in $\mathrm{PBC}$, it seems that they may be independently regulated. This is also supported from clinical data that have shown that AMA titers have no role in disease progression in PBC patients. DC-based immune therapy has been applied in several clinical conditions, either to upregulate host immunity or to down regulate that to achieve therapeutic efficacy. ${ }^{10}$ However, a clinically acceptable protocol is yet to be developed. The 
present study, although a pilot one, has unmasked some important information for containment of hepatic damages in animal model of PBC. Further study using human regulatory DC would unveil the real implications of these observations.

\section{REFERENCES}

1. Kaplan MM, Gershwin ME. Primary biliary cirrhosis. N Engl J Med 2005;353:1261-73.

2. Invernizzi $P$, Selmi $C$, Gershwin ME. Update on primary biliary cirrhosis. Dig Liver Dis 2010;42:401-08.

3. Hohenester S, Oude-Elferink RP, Beuers U. Primary biliary cirrhosis. Semin Immunopathol 2009;31:283-307.

4. Selmi C, Zuin M, Gershwin ME. The unfinished business of primary biliary cirrhosis. J Hepatol Sep 2008;49(3):451-60.

5. Newton JL, Jones DE. Managing systemic symptoms in chronic liver disease. J Hepatol 2012;56(Suppl):S46-55.

6. EASL clinical practice guidelines: Management of cholestatic liver diseases. J Hepatol 2009;51:237-67.

7. Poupon RE, et al. Ten-year survival in ursodeoxycholic acidtreated patients with primary biliary cirrhosis. The UDCA-PBC Study Group. Hepatology 1999;29:1668-71.

8. Goulis J, Leandro G, Burroughs AK. Randomised controlled trials of ursodeoxycholic-acid therapy for primary biliary cirrhosis: A meta-analysis. Lancet 1999;354:1053-60.

9. Iwasaki S, Ohira H, Nishiguchi S, Zeniya M, Kaneko S, Onji $\mathrm{M}$, et al. Study group of intractable liver diseases for research on a specific disease, Health Science Research Grant, Ministry of Health, Labour and Welfare of Japan. The efficacy of ursodeoxycholic acid and bezafibrate combination therapy for primary biliary cirrhosis: A prospective, multicenter study. Hepatol Res 2008;38:557-64.

10. Onji M, Akbar SM (Eds). Dendritic cells in clinics. Tokyo: Spirnger 2008:155-69.

11. Torisu M, Murakami H, Akbar SM, Matsui H, Hiasa Y, Matsuura B, Onji M. Protective role of interleukin-10-producing regulatory dendritic cells against murine autoimmune gastritis. Gastroenterol 2008;43:100-07.

12. Yamanishi H, Murakami H, Ikeda Y, Abe M, Kumagi T, Hiasa $\mathrm{Y}$, et al. Regulatory dendritic cells pulsed with carbonic anhydrase I protect mice from colitis induced by CD4+CD25-T Cells. J Immunol 1 Mar 2012;188(5):2164-72.

13. Diana J, Brezar V, Beaudoin L, Dalod M, Mellor A, Tafuri A, et al. Viral infection prevents diabetes by inducing regulatory $T$ cells through NKT cell-plasmacytoid dendritic cell interplay. J Exp Med 2011; 208:729-45.

14. Kleijwegt FS, Laban S, Duinkerken G, Joosten AM, Zaldumbide A, Nikolic T, Roep BO. Critical role for TNF in the induction of human antigen-specific regulatory $\mathrm{T}$ cells by tolerogenic dendritic cells. J Immunol 2010;185:1412-18.

15. Mukherjee G, Dilorenzo TP. The immunotherapeutic potential of dendritic cells in type 1 diabetes. Clin Exp Immunol. 2010;161:197-207.

16. Zheng X, Suzuki M, Ichim TE, Zhang X, Sun H, Zhu F, et al. Treatment of autoimmune arthritis using RNA interferencemodulated dendritic cells. J Immunol 2010;184:6457-64.

17. Pedersen AW, Claesson MH, Zocca MB. Dendritic cells modified by vitamin D: Future immunotherapy for autoimmune diseases. Vitam Horm 2011;86:63-82.
18. Allam JP, Bieber T, Novak N. Dendritic cells as potential targets for mucosal immunotherapy. Curr Opin Allergy Clin Immunol 2009;9:554-57.

19. Okada C, Akbar SMF, Horiike N, Onji M. Early development of primary biliary cirrhosis in female C57BL/6 mice due to poly I: C administration. Liver International 2005;25:595-603.

20. Sasaki M, Allina J, Odin JA, Thung SN, Coppel R, Nakanuma $\mathrm{Y}$, Gershwin ME. Autoimmune cholangitis in the SJL/J mouse is antigen nonspecific. Dev Immunol 2002;9:103-11.

21. Yamamoto K, Akbar SMF, Masumoto T, Onji M. Increased nitric oxide (NO) production by antigen presenting dendritic cells is responsible for low allogenic mixed leukocyte reaction (MLR) in primary biliary cirrhosis. Clin Exp Immunol 1998; 114:94-101.

22. Akbar SMF, Furukawa S, Nakanishi S, Abe M, Horiike N, Onji M. Therapeutic efficacy and decreased nitrite production by bezafibrate in patients with primary biliary cirrhosis. J Gastroenterol 2005;40:157-63.

23. Akbar SMF, Yamamoto K, Miyakawa H, Ninomiya T, Abe M, Hiasa Y, et al. Peripheral blood T-cell responses to pyruvate dehydrogenase complex in primary biliary cirrhosis: Role of antigen-presenting dendritic cells. Eur J Clin Invest 2001;31: $639-46$.

\section{ABOUT THE AUTHORS}

\section{Sheikh Mohammad Fazle Akbar (Corresponding Author)}

Department of Medical Sciences, Toshiba General Hospital, Higashi Oi 6-3-22, Shinagawa, Tokyo 140-8522, Phone: 81-3-3764-0511 Fax: 81-3-3764-8992, e-mail: shikh.akbar@po.toshiba.co.jo

\section{Shiyi Chen}

Department of Gastroenterology and Metabology, Ehime University Graduate School of Medicine, Ehime, Japan

\section{Mamun Al-Mahtab}

Associate Professor, Department of Hepatology, Bangabandhu Sheikh Mujib Medical University, Dhaka, Bangladesh

\section{Masanori Abe}

Department of Gastroenterology and Metabology, Ehime University Graduate School of Medicine, Ehime, Japan

\section{Osamu Yoshida}

Department of Gastroenterology and Metabology, Ehime University Graduate School of Medicine, Ehime, Japan

\section{Yoshio lkeda}

Endoscopy Center, Ehime University Hospital, Japan

\section{Yoichi Hiasa}

Department of Gastroenterology and Metabology, Ehime University Graduate School of Medicine, Ehime, Japan

\section{Morikazu Onji}

Department of Gastroenterology and Metabology, Ehime University Graduate School of Medicine, Ehime, Japan 